\title{
Reprodução e alimentação de Rhamdia quelen (Quoy \& Gaimard, 1824) em rios do Núcleo Santa Virgínia, Parque Estadual da Serra do Mar, São Paulo, SP
}

\author{
Leandro Muller Gomiero ${ }^{1,2}$, Ursulla Pereira Souza ${ }^{1}$ \& Francisco Manoel de Souza Braga ${ }^{1}$
}

Biota Neotropica v7 (n3) - http://www.biotaneotropica.org.br/v7n3/pt/abstract?article +bn01907032007

\author{
Recebido em 18/06/07 \\ Versão reformulada recebida em 20/08/07 \\ Publicado em 20/09/07
}

${ }^{1}$ Departamento de Zoologia, Instituto de Biociências, Universidade Estadual Paulista - UNESP,

CP 199, CEP 13506-900, Av. 24-A, n. 1515, Rio Claro, SP, Brasil

e-mail:psouza@rc.unesp.br,fmsbraga@rc.unesp.br

${ }^{2}$ Autor para correspondência: Leandro Muller Gomiero,e-mail: leanmg@rc.unesp.br

\begin{abstract}
Gomiero, L.M., Souza, U.P. \& Braga, F.M.S. Reproduction and feeding of Rhamdia quelen (Quoy \& Gaimard, 1824) in rivers of the Santa Virgínia Unit, State Park of the Serra do Mar, São Paulo, SP. Biota Neotrop. Sep/Dez 2007 vol. 7, no. 3 http://www.biotaneotropica.org.br/v7n3/pt/abstract?article+bn01907032007. ISSN 1676-0603.

The frames of this work were determine the reproductive period, fecundity, spawning type and feeding habits of Rhamdia quelen in rivers of the Atlantic Forest in Santa Virgínia Unit, State Park of the Serra do Mar, State of São Paulo. Monthly sampling through January to December 2004 were accomplished in two rivers, Paraibuna and Grande. The alimentary activity, fat accumulation in the visceral cavity and the reproductive period occurred throughout the year probably due to the environmental stability in the area. The diet of this specie was characterized as omnivorous, with tendency to insectivore. Rhamdia quelen was classified as fractional spawning type. The mean absolute fecundity ranged from 27,759.7 to 29,757.3 oocytes in Paraibuna and Grande rivers, respectively.

Keywords: Silver catfish, alimentary activity, fecundity, seasonal, Atlantic Forest.

\section{Resumo}

Gomiero, L.M., Souza, U.P. \& Braga, F.M.S. Reprodução e alimentação de Rhamdia quelen (Quoy \& Gaimard, 1824) em rios do Núcleo Santa Virgínia, Parque Estadual da Serra do Mar, São Paulo, SP. Biota Neotrop. Sep/Dez 2007 vol. 7, no. 3 http://www.biotaneotropica.org.br/v7n3/pt/abstract?article+bn01907032007. ISSN 1676-0603.

O objetivo do trabalho foi determinar a época de reprodução, a fecundidade, o tipo de desova e os aspectos alimentares de Rhamdia quelen em rios da Mata Atlântica no Núcleo Santa Virgínia, Parque Estadual da Serra do Mar, estado de São Paulo. Foram realizadas amostragens mensais de janeiro a dezembro de 2004 em dois rios, Paraibuna e Grande, da bacia do rio Paraíba do Sul. A atividade alimentar, o acúmulo de gordura na cavidade visceral e a época reprodutiva ocorreram ao longo de praticamente todo o período de coleta, possivelmente pela falta de sazonalidade na região. Os itens alimentares foram muito diversos e a dieta foi caracterizada como sendo onívora, com tendência ao consumo de insetos. A desova de $R$. quelen foi do tipo parcelada. A fecundidade média foi de 27.759,7 e de 29.757,3 nos rios Paraibuna e Grande, respectivamente.
\end{abstract}

Palavras-chave: Jundiá, atividade alimentar, fecundidade, sazonalidade, Mata Atlântica. 


\section{Introdução}

A Mata Atlântica é um bioma brasileiro fortemente impactado que vem sendo reduzido desde os tempos coloniais pela extração de essências vegetais até os dias de hoje, pela especulação imobiliária. As características reprodutivas e alimentares apresentadas por sua ictiofauna são pouco conhecidas e de relevante interesse biológico para a conservação das espécies. Este ambiente é pouco marcado pela sazonalidade, sem grandes períodos de seca devido às influências do oceano (Tonhasca Jr. 2005). Muitos rios deste sistema se encontram em adiantado estado de degradação com populações ícticas alteradas ou até empobrecidas devido à poluição, introdução de espécies, desmatamento das florestas ribeirinhas e ao barramento de alguns importantes rios (Hilsdorf \& Petrere Jr. 2002).

Segundo Winemiller (1989), a reprodução em peixes ocorre logo após as chuvas associadas ao aumento de temperatura e dos níveis dos rios e lagos. O período de desova é relacionado com a disponibilidade de alimento para as larvas (Bagenal 1971) e a ovulação ocorre rapidamente em resposta aos fatores exógenos específicos, mas também é regulada pelos fatores endógenos (Stacey 1984).

A fecundidade, o período e o tipo de desova são características específicas essenciais para a manutenção de qualquer espécie de peixe (Vazzoler 1996). Além disso, o conhecimento das táticas e estratégias reprodutivas é elemento imprescindível para nortear as medidas de administração, manejo e preservação da ictiofauna frente aos impactos ocasionados por ações antrópicas como a pesca e a eliminação de áreas de desova e de criadouros (Vazzoler \& Menezes 1992).

Além das características reprodutivas, o conhecimento das interações alimentares entre os peixes e os sistemas ripários é essencial para a reabilitação de ambientes degradados e para prevenir a deterioração das populações de peixes de água doce (Pusey \& Arthington 2003).

O bagre Rhamdia quelen (Siluriformes, Heptapteridae) é uma espécie amplamente distribuída na América do Sul e Central, ao leste dos Andes e entre a Venezuela e o Norte da Argentina (Silfvergrip 1996). Esta espécie representou no ano 2000 cerca de 1,4\% do total de pescado produzido pelo setor aquí́cola brasileiro (Bombardelli et al. 2006), sendo muito importante para a piscicultura da região sul do Brasil (Behr et al. 1999). Este bagre tem hábitos noturnos com preferência por locais calmos e profundos dos rios (Gomes et al. 2000) com seixos grandes ou com troncos submersos (Schulz \& Leuchtenberger 2006). É um bagre bentônico especulador do substrato, alimenta-se de insetos terrestres e aquáticos, crustáceos, restos vegetais, além de peixes como os lambaris e os guarús (Casatti et al. 2001, Baldisserotto \& Radünz Neto 2004, Casatti \& Castro 2006, Oyakawa et al. 2006). As fêmeas atingem comprimentos e pesos superiores aos dos machos (Narahara et al. 1985a), sendo 66,5 cm o comprimento assintótico das fêmeas e $52 \mathrm{~cm}$ o dos machos, podendo alcançar mais de $3 \mathrm{~kg}$ (Gomes et al. 2000).

Muitos dos estudos envolvendo o bagre Rhamdia quelen são direcionados à piscicultura e desenvolvidos com espécimens em tanques de criação. Assim, o objetivo do trabalho foi apresentar a época de reprodução, a fecundidade, o tipo de desova e os aspectos alimentares de $R$. quelen em rios da Mata Atlântica no Núcleo Santa Virgínia, Parque Estadual da Serra do Mar, estado de São Paulo.

\section{Material e Métodos}

Os rios Paraibuna e Grande da bacia do Paraíba do Sul estão inseridos no Núcleo Santa Virgínia, cuja área abrange 16.000 ha nos municípios paulistas de São Luiz do Paraitinga, Natividade da Serra, Cunha e Ubatuba, localizando-se nas coordenadas $23^{\circ} 24^{\prime}$ e $23^{\circ} 17^{\prime} \mathrm{S}$ e $45^{\circ} 03^{\prime} \mathrm{O}$. Seu relevo é fortemente escarpado com vales e vertentes retilíneas, com altitudes que variam entre 860 e 1.500 m, a vegetação é caracterizada por Floresta Ombrófila Densa Montana e manchas descontínuas de floresta em vias de regeneração, além de áreas de silvicultura de eucaliptos abandonados com sub-bosque de nativas, o clima é úmido sujeito à massa tropical Atlântica (Villani 1998).

Os exemplares foram capturados mensalmente de janeiro a dezembro de 2004 com redes de espera (malhas 1,5, 2,0, 2,5, 3, 0, 3,5 e 4,0 cm entre nós adjacentes) com $10 \mathrm{~m}$ de comprimento e 1,5 m de altura, além de covos e peneiras. Após a captura, os exemplares foram mantidos em formalina a $10 \%$. A temperatura $\left({ }^{\circ} \mathrm{C}\right)$ da água foi medida por local e período de coleta.

De cada exemplar capturado foram obtidos o peso total (PT) em gramas, o comprimento total (CT) em centímetros, o comprimento do intestino $(\mathrm{CI})$ em centímetros, o sexo, o grau de repleção do estômago (GR), o grau de gordura acumulada na cavidade visceral (GA) e o estágio de maturação gonadal (EM).

Ao grau de repleção estomacal, que indica o estado de enchimento do estômago, foram atribuídas três categorias: 1 = estômago vazio, 2 = estômago parcialmente cheio e 3 = estômago completamente cheio. Os estômagos foram retirados da cavidade visceral, pesados e colocados em formalina a 5\%. Após alguns dias, o conteúdo estomacal foi transferido para álcool diluído a 70\% (Zavala-Camin 1996). A identificação dos itens alimentares ocorreu até o menor nível taxonômico (Borror \& Delong 1988). Para a análise dos itens alimentares foi utilizado o método de freqüência de ocorrência (Hyslop 1980) que representa a relação, expressa em porcentagem, entre o número de estômagos com determinado tipo de item e o número total de estômagos com alimento. Para a análise da atividade alimentar nos diferentes períodos foi utilizado o índice de repleção estomacal, que é dado pela porcentagem do peso do estômago relativo ao peso total de cada exemplar. A comparação das dietas entre locais (Paraibuna e Grande) foi feita por meio do coeficiente de correlação de classes de Spearman-rs (Fritz 1974, Siegel 1975). O QI (quociente intestinal) é o resultado da divisão entre o comprimento do intestino e o comprimento do peixe, sendo utilizado para relacionar o comprimento relativo do intestino com a dieta. Ao grau de gordura acumulada na cavidade visceral foram atribuídas três categorias: 1 = cavidade vazia, 2 = cavidade com gordura acumulada e 3 = cavidade completamente cheia.

O estádio de maturação de cada exemplar foi determinado sob estereomicroscópio ou quando possível, macroscopicamente, considerando cor, transparência, vascularização superficial e, em ovários, a visualização e aparência dos ovócitos. Ao estádio de maturação foram atribuídas, conforme escala previamente estabelecida, quatro categorias: $\mathrm{A}=$ imaturo, $\mathrm{B}=\mathrm{em}$ maturação ou repouso, $\mathrm{C}=$ maduro e $\mathrm{D}=$ desovado (Vazzoler 1996). Uma tabela de contingência do tipo i $x 2$ (onde i > 2) foi usada para a análise da proporção sexual e o teste $\mathrm{G}$ foi empregado para a análise dos estádios de maturidade (A, B, C e D), graus de repleção e de gordura acumulada (1, 2 e 3) (Vanzolini 1993). O período reprodutivo e as mudanças alimentares foram analisados segundo as variações sazonais das frequiências dos estádios de maturação gonadal, de repleção dos estômagos e de gordura acumulada durante o período das coletas.

Para o estudo de fecundidade, segundo Vazzoler (1996), os ovários em estádio $\mathrm{C}$ foram retirados da cavidade visceral e pesados, depois suas membranas foram seccionadas, longitudinalmente, em toda a sua extensão e imersos em solução de Gilson modificada. Estes frascos foram agitados periodicamente, e depois da total dissociação, os ovócitos foram lavados várias vezes em álcool 70\%, onde foram mantidos. A fecundidade foi estimada segundo o método volumétrico (Vazzoler 1996). A fecundidade individual foi estimada considerando $\mathrm{N}^{\prime}=\mathrm{Sn} / \mathrm{s}$, sendo $\mathrm{S}$ o volume de álcool utilizado para homogeneizar a massa de ovócitos, n o número total de ovócitos contados na amostra de volume s obtida pela pipeta Stempel. Assim, estimou-se o número total de ovócitos, N', contidos no par de ovários 
dissociados. Como a fecundidade individual é o número de ovócitos maduros e não o total de ovócitos contido nos ovários, foi utilizada a expressão N = N'.P/100 para estimar a fecundidade individual, sendo $\mathrm{P}$ a porcentagem de ovócitos nos ovários em relação ao número total de ovócitos (N'), a partir do diâmetro em que os ovócitos iniciam o processo de amadurecimento. Os grupos de freqüência de ocorrência por classes de diâmetros dos ovócitos foram determinados segundo o método apresentado em Vazzoler (1996).

Uma vez estimada a fecundidade individual, foi estimada também a fecundidade relativa, em termos de comprimento (FRC) e de peso (FRP). A fecundidade relativa foi estimada dividindo-se o valor da fecundidade individual pelo comprimento (FRC) ou pelo peso (FRP) do respectivo exemplar.

\section{Resultados}

Foram capturados 74 exemplares de $R$. quelen ao longo dos 12 meses de estudo. A proporção sexual foi equilibrada, sem diferenças significativas $\left(\chi^{2}>0,05\right)$ entre as amostras de peixes dos rios Paraibuna e Grande. Apesar disso, no rio Grande apenas fêmeas foram capturadas durante o outono e apenas machos durante o inverno (Figura 1). A distribuição das frequiências de machos e fêmeas nas classes de comprimento total $(\mathrm{cm})$ variou de 13 a $30,9 \mathrm{~cm}$, com maior freqüência na classe de 21 a 22,9 $\mathrm{cm}$ para ambos sexos. A análise com o teste Kolmogorov-Smirnov da distribuição das freqüências nas classes de comprimento total mostrou que não houve diferença significativa $\left(\chi^{2}>0,05\right)$ entre as distribuições de machos e fêmeas. Porém, houve uma tendência dos machos nas menores classes de comprimentos e de fêmeas nas maiores classes (Figura 2).

As análises dos graus de repleção estomacal não indicaram diferenças sazonais significativas $\left(\chi^{2}>0,05\right)$ tanto no rio Paraibuna quanto no rio Grande. O grau de repleção estomacal 3 (repleto) ocorreu em todos os períodos, exceto no outono em ambos os rios abordados. O grau 2 (parcialmente repleto) foi bem representativo em todas as estações, sendo mais freqüente na primavera. Houve uma grande quantidade de estômagos vazios $(\mathrm{GR}=1)$ no rio Grande, durante o verão (Figura 3). Com a análise do índice de repleção estomacal observou-se que em todos os períodos ocorreu considerável atividade alimentar, sendo maior na primavera e verão no rio Paraibuna e no inverno no rio Grande (Figura 4).

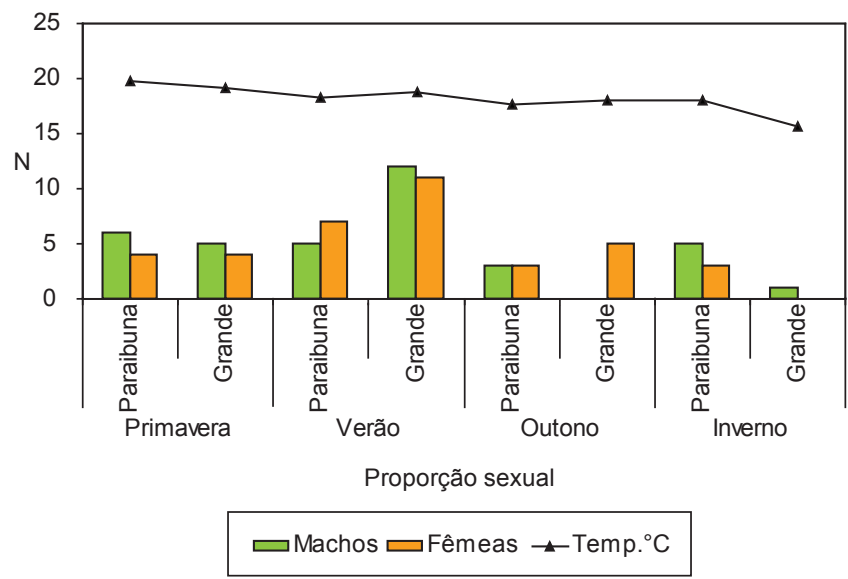

Figura 1. Distribuição numérica da proporção sexual de Rhamdia quelen e temperatura média da água por local e período de coleta.

Figure 1. Numerical distribution of Rhamdia quelen by sex, and temperature in each site and period of sample.

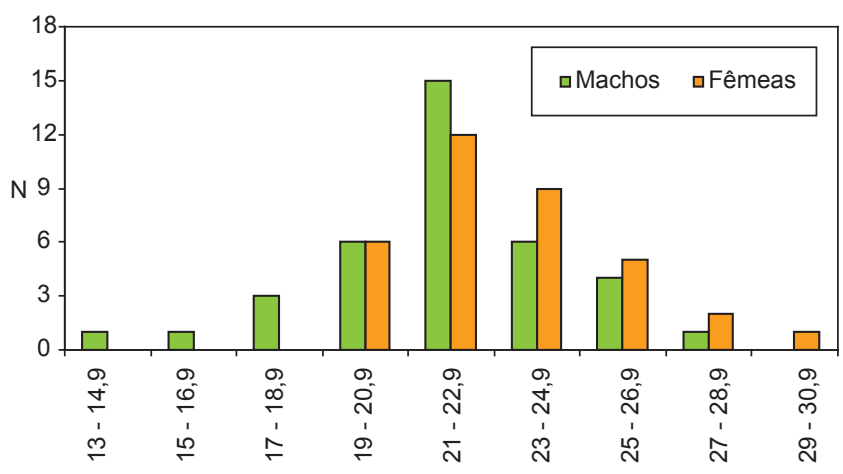

Classes de comprimento $(\mathrm{cm})$

Figura 2. Distribuição nas classes de comprimento de Rhamdia quelen para machos e fêmeas.

Figure 2. Length distribution of Rhamdia quelen for males and females.

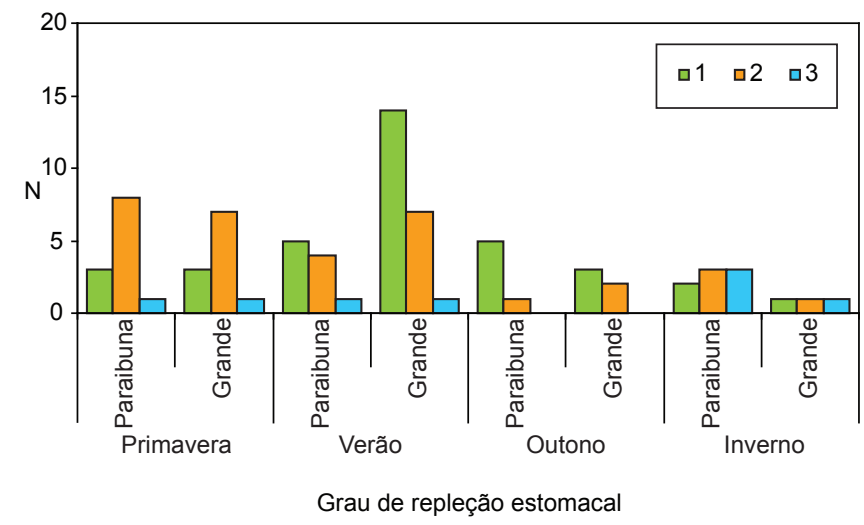

Figura 3. Distribuição numérica do grau de repleção estomacal (1. vazio, 2. parcialmente cheio e 3. cheio) de Rhamdia quelen por período e em cada local de amostragem.

Figure 3. Numerical distribution of the repletion degree of the stomach (1. empty, 2. partly full, 3. full) of Rhamdia quelen per period and in each sampling locality.

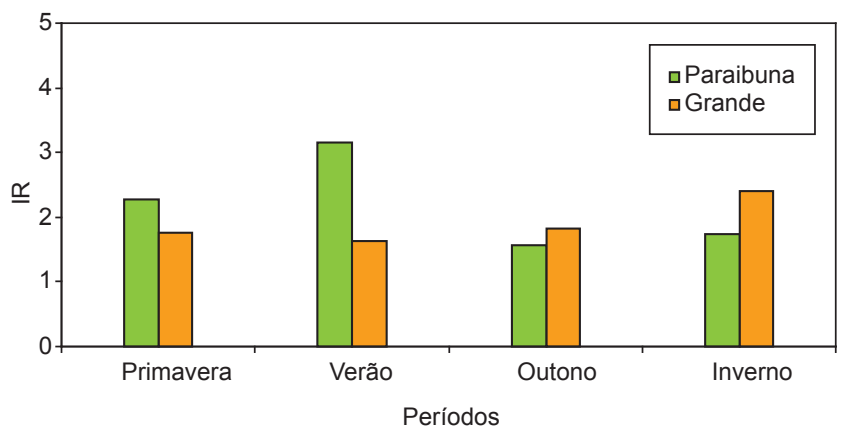

Figura 4. Índice de repleção estomacal por período e em cada local de amostragem.

Figure 4. Repletion indexes of the stomach per period and in each sampling locality. 
As análises dos graus de gordura acumulada na cavidade visceral também não indicaram diferenças sazonais significativas $\left(\chi^{2}>0,05\right)$ em ambos os rios, porém, o grau 3 (repleto) ocorreu principalmente nos exemplares do rio Grande durante a primavera e verão (Figura 5).

A média do quociente intestinal (QI) para os indivíduos do rio Paraibuna foi de 0,83 e do rio Grande foi de 0,75 . O quociente intestinal dos machos foi de 0,76 e das fêmeas foi de 0,81 . Os exemplares de 14 a $18 \mathrm{~cm}$ de comprimento total apresentaram QI de 0,65 , valor inferior às demais classes de comprimentos totais (Tabela 1).

Os itens alimentares consumidos por $R$. quelen nos dois rios amostrados foram Coleoptera, Hymenoptera, Trycoptera, Diptera, Hemiptera, Odonata, Blattaria, Ephemeroptera, Orthoptera, Escamas, Peixes, Nematoda, Crustacea, Diplopoda, Mollusca, Annelida, material vegetal, restos de insetos e sedimentos. Por meio da análise da frequiência de ocorrência foi possível evidenciar que os insetos foram muito importantes para a alimentação da espécie, porém os itens de origem vegetal também foram freqüentes na alimentação dos peixes em ambos ambientes. Os itens Hymenoptera, Blattaria, Escamas e Diplopoda ocorreram apenas nos estômagos dos exemplares do rio Paraibuna; por outro lado, Orthoptera, Peixes e Crustacea ocorreram somente na dieta dos exemplares do rio Grande (Figura 6).

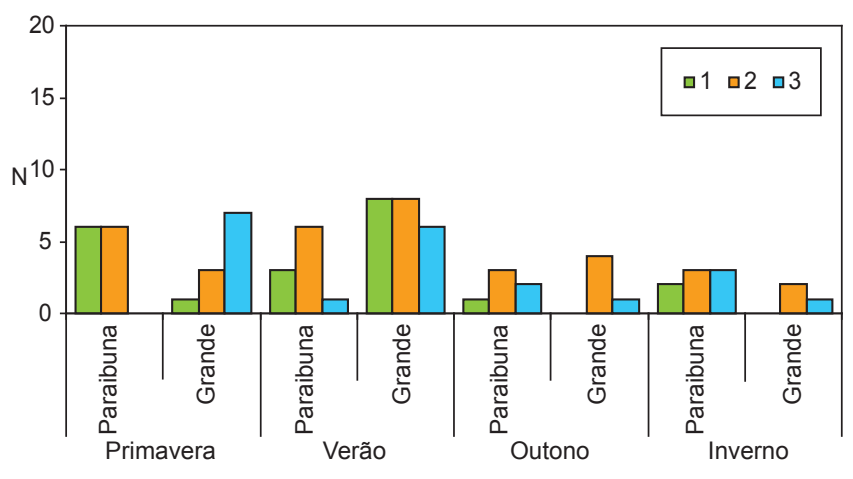

Gordura acumulada

Figura 5. Distribuição numérica do grau de gordura acumulada na cavidade visceral (1. vazio, 2. parcialmente cheio e 3. cheio) de Rhamdia quelen por período e em cada local de amostragem.

Figure 5. Numerical distribution of the fat degree in the visceral cavity (1. empty, 2. partly full, 3. full) of Rhamdia quelen per period and in each sampling locality.

Tabela 1. Quociente intestinal (QI) para Rhamdia quelen nos rios Paraibuna e Grande para machos, fêmeas e por classe de comprimentos.

Table 1. Intestinal quotient (IQ) of Rhamdia quelen in the rivers Paraibuna and Grande for males, females and by length classes.

\begin{tabular}{cccc}
\hline Locais & $\mathbf{N}$ & QI $($ Média \pm DP) & Intervalo \\
\hline Paraibuna & 32 & $0,83 \pm 0,22$ & $(1,69-0,42)$ \\
Grande & 39 & $0,75 \pm 0,13$ & $(0,99-0,48)$ \\
\hline Sexo & $\mathbf{N}$ & QI (Média \pm DP) & Intervalo \\
\hline Machos & 36 & $0,76 \pm 0,19$ & $(1,69-0,42)$ \\
Fêmeas & 35 & $0,81 \pm 0,17$ & $(1,12-0,48)$ \\
\hline Comp. (cm) & $\mathbf{N}$ & QI (Média \pm DP) & Intervalo \\
\hline $14 \mid-18$ & 3 & $0,65 \pm 0,08$ & $(0,75-0,60)$ \\
$18 \mid-22$ & 27 & $0,75 \pm 0,13$ & $(1,12-0,49)$ \\
$22 \mid-26$ & 34 & $0,82 \pm 0,20$ & $(1,69-0,48)$ \\
$26 \mid-30$ & 7 & $0,82 \pm 0,20$ & $(1,01-0,42)$ \\
\hline
\end{tabular}

O teste " $t$ " para o $r_{s}$ de 0,445 indicou que a correlação não foi significativa $\left(\mathrm{t}=2,04 ; \mathrm{t}_{0,05: 17}=2,11 ; \mathrm{p}>0,05\right)$. Portanto, as dietas de $R$. quelen nos dois locais de coletas foram quantitativamente diferentes.

Os estádios de maturidade gonadal de exemplares do rio Paraibuna exibiram diferenças sazonais significativas $\left(\chi^{2}<0,05\right)$, ao contrário daqueles de exemplares procedentes do rio Grande $\left(\chi^{2}>0,05\right)$. Na maioria das estações do ano foram encontrados exemplares com gônadas maduras (estádio C), com exceção do outono (rio Paraibuna) e inverno (rio Grande). A maior freqüência de gônadas maduras ocorreu na primavera e verão, sendo bastante elevada no verão, principalmente no rio Grande. O estádio D (desovado) foi observado no verão e no outono, apenas nos exemplares amostrados no rio Grande e não foram amostrados indivíduos em estádio imaturo (A) em ambos locais de coleta. O período de reprodução da espécie na área de estudo é longo, tendo picos na primavera e verão (Figura 7).

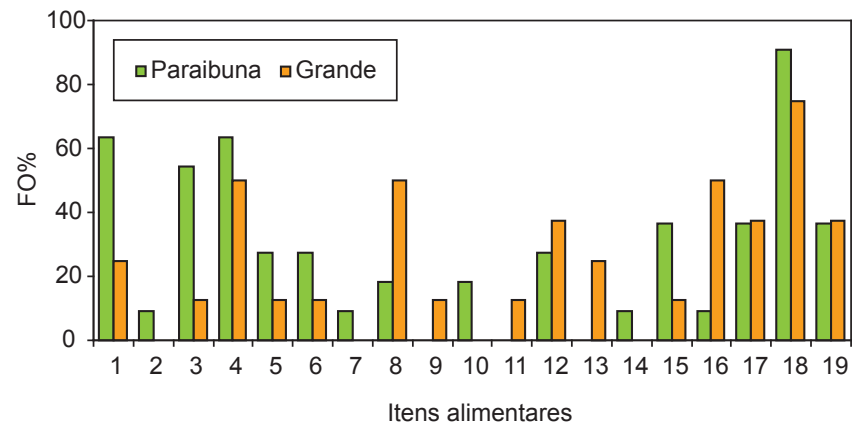

Figura 6. Freqüência de ocorrência dos itens alimentares consumidos por Rhamdia quelen nos rios Paraibuna e Grande: 1. Coleoptera, 2. Hymenoptera, 3. Trycoptera, 4. Diptera, 5. Hemiptera, 6. Odonata, 7. Blattaria, 8. Ephemeroptera, 9. Orthoptera, 10. Escamas, 11. Peixes, 12. Nematoda, 13. Crustacea, 14. Diplopoda, 15. Mollusca, 16. Annelida, 17. Material Vegetal, 18. Restos de insetos, 19. Sedimentos.

Figure 6. Frequency of occurrence of the food items consumed by Rhamdia quelen in the Paraibuna and Grande rivers: 1. Coleoptera, 2. Hymenoptera, 3. Trychoptera, 4. Diptera, 5. Hemiptera, 6. Odonata, 7. Blattaria, 8. Ephemeroptera, 9. Orthoptera, 10. Scales, 11. Fish, 12. Nematoda, 13. Crustacea, 14. Diplopoda, 15. Mollusca, 16. Annelida, 17. Vegetal remains, 18. Insect remains, 19. Sediments.

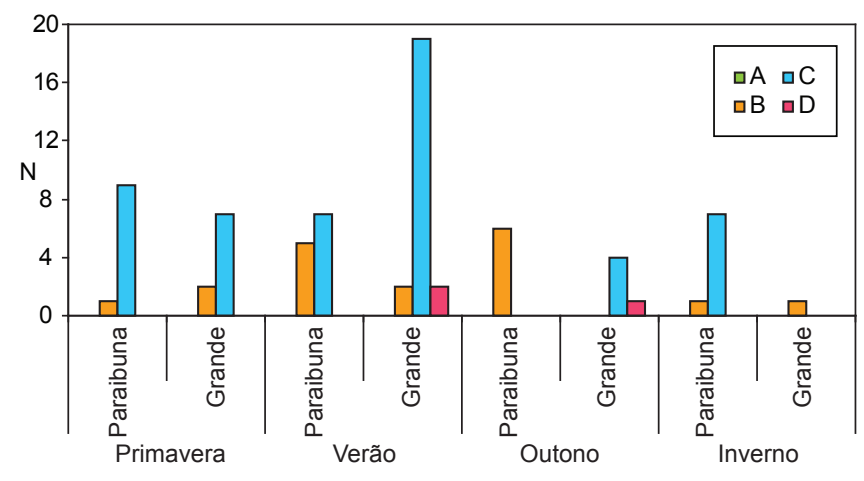

Estádios de maturidade gonadal

Figura 7. Distribuição numérica dos estádios de maturidade gonadal (A. imaturos, B. em maturação, C. maduros, D. desovados) de Rhamdia quelen por período e em cada local de amostragem.

Figure 7. Numerical distribution of the stages of gonadal maturity in Rhamdia quelen (A. immature, B. in maturation, C. mature, D. spent) per period and in each sampling locality. 
Foram analisados 12 pares de ovários provenientes de exemplares do rio Paraibuna, o número total de ovócitos (N') variou de 29.100 a 108.500 ovócitos, com média de 50.347,9 \pm 6.425,9 (Erro da média), este valor não corresponde à fecundidade, pois engloba os ovócitos do lote estoque, em maturação e maduros. A fecundidade $(\mathrm{N})$ variou de 14.775 a 69.440 ovócitos, com média de 27.759,7 $\pm 4.262,7$ (E.M). A média da FRC (fecundidade relativa ao comprimento) foi de 1.154,8 $\pm 205,8$ (E.M) e a média da FRP (fecundidade relativa ao peso) foi de $204 \pm 44,4$ (E.M). Para o rio Grande foram analisados 17 pares de ovários, o número total de ovócitos (N') variou de 23.150 a 87.975 ovócitos, com média de 52.569,1 \pm 4.729 (Erro da média). A fecundidade $(\mathrm{N})$ variou de $9.120,5$ a 54.080 ovócitos, com média de 29.757,3 \pm 3.065,6 (E.M). A média da FRC foi de 1.286,1 \pm 127 (E.M) e a média da FRP foi de 256,9 $\pm 22,6$ (E.M). O tamanho que os ovócitos tornam-se maduros foi de 795,6 $\mu \mathrm{m}$ atingindo diâmetro máximo com 1.162,8 $\mu \mathrm{m}$. O lote estoque abrange ovócitos de 61,2 a $306 \mu \mathrm{m}$. A única diferença dos grupos de frequiência de ocorrência por classes de diâmetros dos ovócitos entre os dois locais amostrados foi que no rio Paraibuna foram evidenciados cinco grupos e no rio Grande, seis grupos (Figuras 8 e 9). A análise destes grupos demonstra que na área de estudo a desova de $R$. quelen é do tipo parcelada.
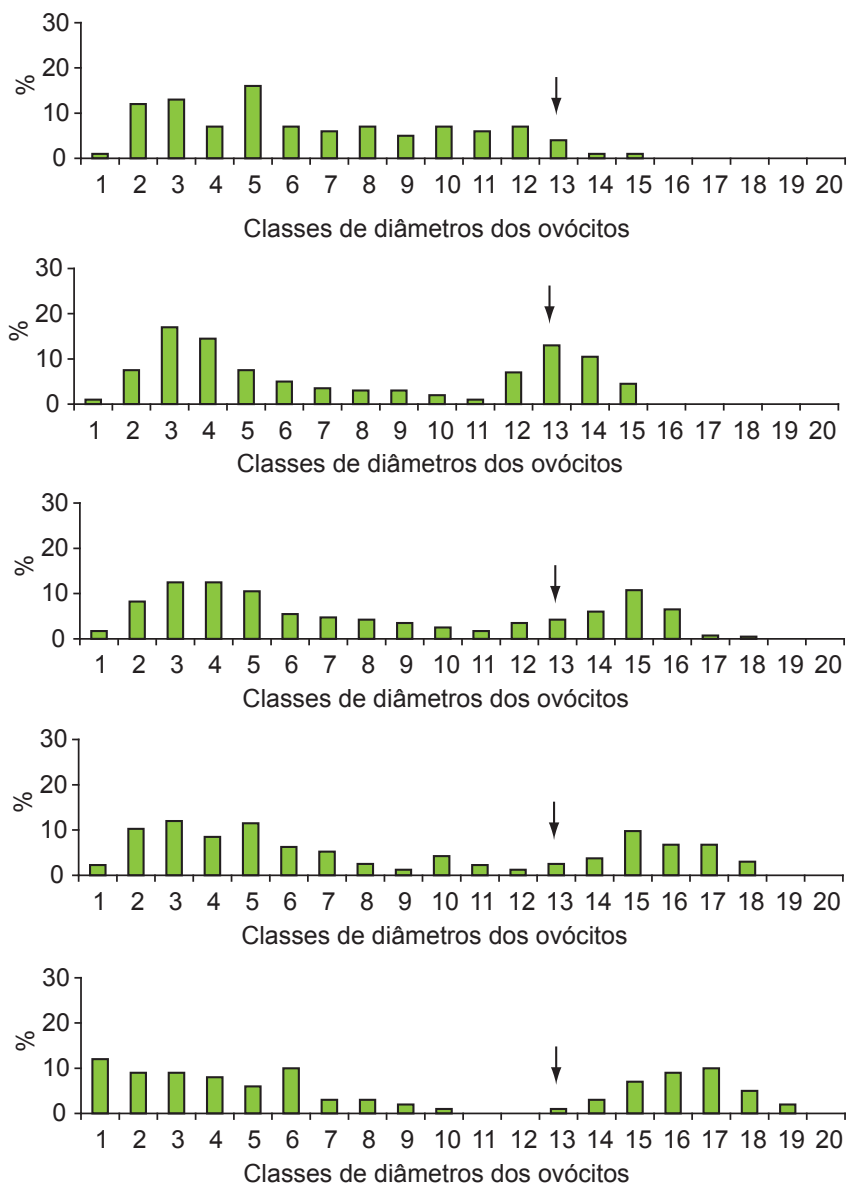

Figura 8. Freqüência porcentual das classes de diâmetro dos ovócitos em d.o.m. (divisão de ocular micrométrica) equivalente a 61,2 micrômetros, de Rhamdia quelen para o rio Paraibuna, divididos em grupos. As setas indicam o tamanho a partir do qual os ovócitos se tornam maduros.

Figure 8. Frequency (in percentage) of the diametrical classes of oocytes, in d.o.m. (division of ocular micrometer) equivalent to 61.2 micrometer, of Rhamdia quelen for the Paraibuna river divided into groups. Arrows indicate the size above which the oocytes become mature.

\section{Discussão}

A falta de sazonalidade bem marcada em algumas regiões de mata Atlântica (Tonhasca Jr. 2005) pode ter sido o fator determinante da atividade alimentar e do acúmulo de gordura na cavidade visceral terem ocorrido ao longo de praticamente todo o período das coletas. Rezende \& Mazzoni (2005) estudando outra área de mata Atlântica verificaram um maior aporte de material proveniente da vegetação ribeirinha durante a estação chuvosa, tanto de itens vegetais quanto de insetos, que podem ser consumidos por peixes que habitam essas áreas.

As informações dos valores de QI são muito úteis para a averiguação das variações do comportamento alimentar entre os indivíduos de uma mesma espécie por ponto de coleta, entre os sexos e nas classes de comprimentos totais (Zavala-Camin 1996). Os valores de QI apresentados por $R$. quelen nos dois rios, entre os sexos e entre as classes de comprimentos totais evidenciam uma dieta onívora com uma tendência de maior ingestão de itens de origem animal. Para $R$. quelen os valores de QI tiveram poucas oscilações, entretanto,
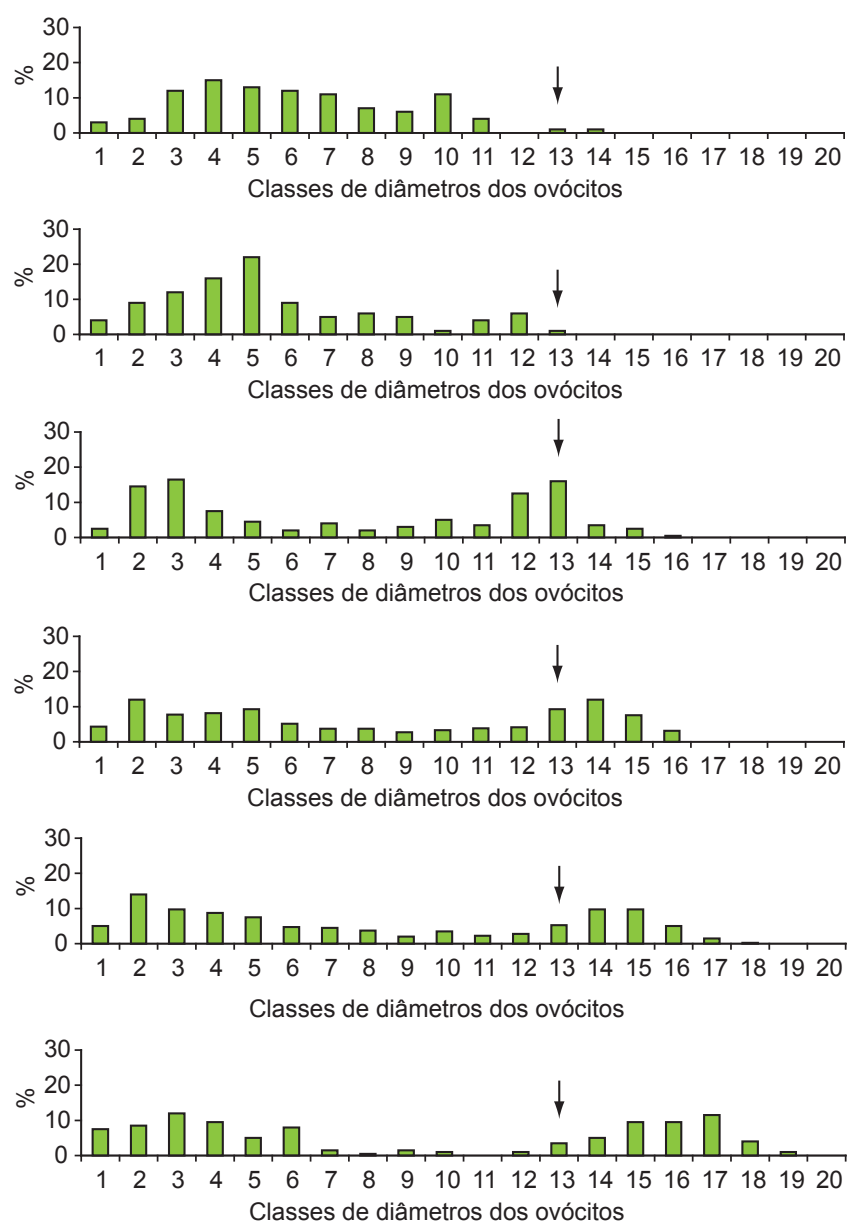

Figura 9. Frequiência porcentual das classes de diâmetro dos ovócitos em d.o.m. (divisão de ocular micrométrica) equivalente a 61,2 micrômetros, de Rhamdia quelen para o rio Grande, divididos em grupos. As setas indicam o tamanho a partir do qual os ovócitos se tornam maduros.

Figure 9. Frequency (in percentage) of the diametrical classes of oocytes, in d.o.m. (division of ocular micrometer) equivalent to 61.2 micrometer, of Rhamdia quelen for the Grande river divided into groups. Arrows indicate the size above which the oocytes become mature. 
foram típicos de espécies onívoras e generalistas (Menin \& Mimura 1992), sendo notória a importância de insetos e material vegetal. Os rios margeados por vegetação nativa fornecem grande variedade de itens alimentares, principalmente insetos e vegetais superiores, que são de grande importância na sobrevivência e no comportamento de peixes tropicais de água doce (Lowe-McConnell 1999). Esta variedade de itens alimentares justifica o predomínio de espécies de peixes generalistas e oportunistas que mudam de um tipo de alimento para outro, tão logo ocorram oscilações na abundância relativa do recurso alimentar em uso (Abelha et al. 2001). Gomes et al. (2000) caracterizaram $R$. quelen como onívora com tendência à piscivoria. A baixa diversidade de peixes de pequeno porte na área de estudo (Gomiero \& Braga 2006), que potencialmente seriam predados por $R$. quelen, pode ter influenciado na ausência do item peixes na dieta, principalmente no rio Paraibuna.

A diferença das dietas apresentadas pelos peixes dos dois rios (Paraibuna e Grande) foi principalmente quantitativa em relação às Ordens dos insetos consumidos. Os itens alimentares autóctones a alóctones são dependentes da mata ribeirinha (Alvim \& Peret 2004) e a conservação destas áreas garante o fornecimento de grande variedade de itens alimentares para a ictiofauna (Lowe-McConnell 1999), podendo ocorrer variações espaço-temporais na disponibilidade destes itens (Abelha et al. 2001).

Constatou-se para os exemplares de R. quelen de outras localidades que indivíduos de um ano de idade com comprimentos acima de $13,4 \mathrm{~cm}$ (machos) e $14 \mathrm{~cm}$ (fêmeas) estavam aptos para a reprodução, com as gônadas em maturação ou maduras (Narahara et al. 1985a, Gomes et al. 2000, Baldisserotto \& Radünz Neto 2004). Todos os indivíduos capturados na área de estudo apresentavam comprimentos superiores aos citados, fazendo parte da parcela reprodutiva da população. Isto pode ser reflexo da seletividade dos aparelhos de pesca utilizados ou que a área amostrada não abriga indivíduos imaturos da população. Lemes \& Garutti (2002) coletaram exemplares de Rhamdia hilarii ( $=R$. quelen) com até $10 \mathrm{~cm}$ de comprimento total em áreas de cabeceiras, tanto em águas rápidas quanto nos poções, portanto os imaturos desta espécie preferiram ambientes distintos dos que foram amostrados neste estudo.

A tendência dos maiores tamanhos das fêmeas em relação aos machos é comum para peixes. O maior tamanho acarreta em maior fecundidade e assim em maior sucesso reprodutivo (Conover 1984, Vazzoler 1996). A fecundidade aumenta proporcionalmente aos incrementos em peso e comprimento, então quanto maior a fêmea, mais ovócitos serão produzidos (Lowe-McConnell 1999).

Exemplares de $R$. quelen apresentaram gônadas maduras durante todos os períodos, com maior intensidade na primavera e verão. Esta tendência de período reprodutivo longo com uma parcela da população sempre pronta para a reprodução foi registrada em outras espécies (Brycon opalinus e Oligosarcus hepsetus) nos mesmos locais (L.M. Gomiero \& F.M.S. Braga, dados não publicados). O período reprodutivo prolongado ocorre em ambientes relativamente estáveis; por outro lado, em ambientes onde a sazonalidade reprodutiva é pronunciada ocorrem maiores flutuações na disponibilidade de alimento ou na competição interespecíficas por locais de desova (Kramer 1978). A reprodução desta espécie foi abordada em diversos locais com época reprodutiva restrita a primavera e verão (Narahara et al. 1985b, Silveira et al. 1985, Gomes et al. 2000, Baldisserotto \& Radünz Neto 2004, Oyakawa et al. 2006). Nos llanos da Venezuela, $R$. quelen apresentou estratégia sazonal que é explicada pelas variações temporais e espaciais na qualidade dos hábitats para o desenvolvimento e sobrevivência dos juvenis (Winemiller 1989). Possivelmente, estes ambientes apresentaram maior sazonalidade em comparação aos da Mata Atlântica, que não tem um período caracteristicamente seco, devido às influências do oceano (Tonhasca Jr. 2005).
A espécie é ovulípara com ovos esféricos, demersais e nãoadesivos. Existe um sincronismo entre machos e fêmeas durante a reprodução, que ocorre logo ao amanhecer, o macho segue a fêmea e promove alguns toques com a cabeça no seu ventre estimulando a desova (Baldisserotto \& Radünz Neto 2004). Não apresenta cuidado parental e desova em locais com água limpa, calma e de fundo pedregoso (Godinho et al. 1978, Gomes et al. 2000, Pereira et al. 2006). Os valores encontrados para a FRP (fecundidade relativa ao peso) de $R$. quelen nos rios Paraibuna e Grande foram próximos do apresentado para Rhamdia branneri $(=R$. quelen) em ambientes naturais preservados do Paraná (Paula-Souza 1978 apud Gomes et al. 2000), mas foi superior ao de $R$. quelen em confinamento e inseminados artificialmente (Bombardelli et al. 2006). A fecundidade é uma adaptação específica para condições variáveis, especialmente para a mortalidade, que se altera primariamente em resposta ao suprimento alimentar e isto é um dos mecanismos básicos para ajustar a taxa de reprodução para as mudanças das condições ambientais. Estas alterações são refletidas nas diferentes fecundidades entre populações e entre espécies (Galvani \& Coleman 1998).

Narahara et al. (1989) determinaram para $R$. hilarii (= R. quelen) os tamanhos em que os ovócitos estavam maduros, variando de 500,8 a 1.377,2 $\mu \mathrm{m}$, o que não diferiu do apresentado pelos exemplares de R. quelen dos rios Paraibuna e Grande.

A desova do tipo parcelada ou múltipla para $R$. quelen foi comum nos mais variados ambientes (Narahara et al. 1989, Winemiller 1989, Gomes et al. 2000, Baldisserotto \& Radünz Neto 2004). Além disso, a divisão em 5 ou 6 grupos de desenvolvimento ovocitário também foi freqüente (Cussac \& Maggese 1986). Em espécies de peixes com período reprodutivo longo a desova parcelada é comum e garante maior suprimento alimentar para as larvas (Vazzoler 1996).

Em um ambiente estável e previsível como o da Mata Atlântica, onde a sazonalidade não é marcada e não existe uma época determinada de cheia ou seca, os recursos alimentares parecem ser mais constantes do que em outros ambientes, sem nenhum acréscimo ou decréscimo pronunciado. As atividades alimentar e reprodutiva aparentemente foram contínuas, mesmo que ocorressem épocas de maior ou menor intensidade. As características similares para estes parâmetros em diferentes espécies de peixes desta área (Brycon opalinus e Oligosarcus hepsetus) apontam para uma tendência comum determinada pelos aspectos ambientais. Os valores de fecundidade, FRC (fecundidade relativa ao comprimento), FRP (fecundidade relativa ao peso) e de tamanho dos diâmetros de ovócitos maduros apresentados por $R$. quelen não tiveram diferenças marcantes entre os mais variados ambientes e os encontrados na área de estudo. Estes fatores podem ser menos mutáveis sob a influência da sazonalidade do ambiente e serem determinados principalmente pela mortalidade.

\section{Agradecimentos}

O autor (L.M.G.) agradece à FAPESP (proc.03/05696-1), à Cotec (proc.40.673/03) e ao IBAMA (aut.055/2003).

\section{Referências Bibliográficas}

ABELHA, M.C.F., AGOSTINHO, A.A. \& GOULART, E. 2001. Plasticidade trófica em peixes de água doce. Acta Scientiarum 23(2):425-434.

ALVIM, M.C.C. \& PERET, A.C. 2004. Food resources sustaining the fish fauna in a section of the upper São Francisco River in Três Marias, MG, Brazil. Braz. J. Biol. 64(2):195-202.

BAGENAL, T.B. 1971. The interrelation of the size of fish eggs, the date of spawning and the production cycle. J. Fish Biol. 3:207-219.

BALDISSEROTTO, B. \& RADÜNZ NETO, J. 2004. Criação de Jundiá. Ed. UFSM, Santa Maria. 
BEHR, E.R., NETO, J.R., TRONCO, A.P. \& FONTANA, A.P. 1999. Influência de diferentes níveis de luminosidade sobre o desempenho de larvas de Jundiá (Rhamdia quelen) (Quoy \& Gaimard, 1824) (Pisces: pimelodidae). Acta Scientiarum 21(2):325-330.

BOMBARDELLI, R.A., MÖRSCHBÄCHER, E.F., CAMPAGNOLO, R., SANCHES, E.A. \& SYPERRECK, M.A. 2006. Dose inseminante para fertilização artificial de ovócitos de jundiá cinza, Rhamdia quelen (Quoy \& Gaimard, 1824). R. Bras. Zootec. 35(4):1251-1257.

BORROR, D.J. \& DELONG, D.M. 1988. Introdução ao estudo dos insetos. Editora Edgard Blucher, São Paulo.

CASATTI, L. \& CASTRO, R.M.C. 2006. Testing the ecomorphological hypothesis in a headwater riffles fish assemblage of the rio São Francisco, southeastern Brazil. Neotrop. Ichthyol. 4(2):203-214.

CASATTI, L., LANGEANI, F. \& CASTRO, R.M.C. 2001. Peixes de riacho do Parque Estadual Morro do Diabo, bacia do alto rio Paraná, SP. Biota Neotrop. 1:1-15

CONOVER, D.O. 1984. Adaptive significance of temperature-dependent sex determination in a fish. Am. Nat. 123(03):297-313.

CUSSAC, V.E. \& MAGGESE, M.C. 1986. Oogenesis in Rhamdia sapo (Pisces, Pimelodidae). Stages of the oocyte, egg's envelopes and effects of the human chorionic gonadotropin. Rev. Brasil. Biol. 46(1):139-147.

FRITZ, E.S. 1974. Total diet comparison in fishes by Spearman rank correlation coefficients. Copeia 1:210-214.

GALVANI, A.P. \& COLEMAN, R.M. 1998. Do parental convict cichlids of different sizes value the same brood number equally? Animal Behaviour 56:541-546.

GODINHO, H.M., FENERICH, N.A. \& NARAHARA, N.Y. 1978. Desenvolvimento embrionário e larval de Rhamdia hilarii (Valenciennes, 1840) (Siluriformes, Pimelodidae). Rev. Brasil. Biol. 38(1):151-156.

GOMES, L.C., GOLOMBIESKI, J.I., GOMES, A.R.C. \& BALDISSEROTTO, B. 2000. Biologia do jundiá Rhamdia quelen (Teleostei, Pimelodidae). Ciência Rural 30(1):179-185.

GOMIERO, L.M. \& BRAGA, F.M.S. 2006. Diversity of the ichthyofauna in the Serra do Mar State Park- Núcleo Santa Virgínia, São Paulo State, Brazil. Acta Scientiarum 28(3):213-218.

HILSFORF, A.W.S. \& Petrere Jr., M. 2002. Conservação de peixes na bacia do rio Paraíba do Sul. Ciência Hoje 30(180):62-65.

HYSLOP, E.J. 1980. Stomach contents analysis: a review of methods and their application. J. Fish Biol. 17(4):411-429.

KRAMER, D.L. 1978. Reproductive seasonality in the fishes of a tropical stream. Ecology 59(5):976-985.

LEMES, E.M. \& GARUTTI, V. 2002. Ictiofauna de poção e rápido em um córrego de cabeceira da bacia do Alto rio Paraná. Comun. Mus. Ciênc. Tecnol. 15(2):175-199.

LOWE-McCONNELL, R.H. 1999. Estudos ecológicos de comunidades de peixes tropicais (USP, ed.). São Paulo.

MENIN, E. \& MIMURA, O.M. 1992. Anatomia funcional comparativa do intestino de duas espécies de peixes teleostei de hábitos alimentares distintos. Rev. Ceres 39(22):308-341.

NARAHARA, M.Y., GODINHO, H.M., FENERICH-VERANI, N. \& ROMAGOSA, E. 1985a. Relação peso-comprimento e fator de condição de Rhamdia hilarii (Valenciennes, 1840) (Osteichthyes, Siluriformes, Pimelodidae). B. Inst. Pesca 12(4):13-22.

NARAHARA, M.Y., GODINHO, H.M. \& ROMAGOSA, E. 1985b. Estrutura da população de Rhamdia hilarii (Valenciennes, 1840) (Osteichthyes, Siluriformes, Pimelodidae). B. Inst. Pesca 12(3):123-137.

NARAHARA, M.Y., GODINHO, H.M. \& ROMAGOSA, E. 1989. Tipo de desova e fecundidade do bagre, Rhamdia hilarii (Valenciennes, 1840) (Siluriformes, Pimelodidae). B. Inst. Pesca 16(1):37-45.

OYAKAWA, O.T., AKAMA, A., MAUTARI, K.C. \& NOLASCO, J.C. 2006. Peixes de riachos da Mata Atlântica. Ed. Neotrópica, São Paulo.

PEREIRA, C.R., BARCELLOS, L.J.G., KREUTZ, L.C., QUEVEDO, R.M., RITTER, F. \& SILVA, L.B. 2006. Embryonic and larval development of jundiá (Rhamdia quelen, Quoy \& Gaimard, 1824, Pisces, Teleostei), a south american catfish. Braz. J. Biol. 66(4):1057-1063.

PUSEY, B.J. \& ARTHINGTON, A.H. 2003. Importance of the riparian zone to the conservation and management of freshwater fish: a review. Mar. Freshw. Res. 54:1-16.

REZENDE, C.F. \& MAZZONI, R. 2005. Seasonal variation in the input of allochthonous matter in an Atlantic Rain Forest stream, Ilha Grande-RJ. Acta Limnol. Bras. 17(2):167-175.

SCHULZ, U.H. \& LEUCHTENBERGER, C. 2006. Activity patterns of South American silver catfish (Rhamdia quelen). Braz. J. Biol. $66(2 \mathrm{~A}): 565-574$

SIEGEL, S. 1975. Estatística não-paramétrica para as ciências do comportamento. McGraw-Hill do Brasil, Rio de Janeiro.

SILVEIRA, W.F., KAVAMOTO, E.T. \& NARAHARA, M.Y. 1985. Avaliação da qualidade e crio-preservação em forma de "pellets" do sêmen do bagre, Rhamdia hilarii (Valenciennes, 1840). B. Inst. Pesca 12(4):7-11.

SILFVERGRIP, A.M.C. 1996. A sistematic revision of the neotropical catfish genus Rhamdia (Teleostei, Pimelodidae). PhD Thesis, Stockholm University and Department of Vertebrate Zoology, Stockholm, Swedem.

STACEY, N.E. 1984. Control of timing of ovulation by exogenous and endogenous factors. In Fish reproduction: strategies and tactics (Potts, G. W. \& Wooton, R. J., ed.). Londres, v. 12, p. 207-222.

TONHASCA JR., A. 2005. Ecologia e história natural da Mata Atlântica. Ed. Interciência, Rio de Janeiro.

VANZOLINI, P.E. 1993. Métodos estatísticos elementares em sistemática zoológica. Ed. HUCITEC, São Paulo.

VAZZOLER, A.E.A.M. 1996. Biologia da reprodução de peixes teleósteos: Teoria e Prática. Eduem, Maringá.

VAZZOLER, A.E.A.M. \& MENEZES, N.A. 1992. Síntese de conhecimentos sobre o comportamento reprodutivo dos Characiformes da América do Sul (Teleostei, Ostariophysi). Rev. Brasil. Biol. 52(4):627-640.

VILLANI, J.P. 1998. Plano de manejo das unidades de conservação: Parque Estadual da Serra do Mar-Núcleo Santa Virgínia. Plano de Gestão Ambiental-Fase 1. Secretaria do Meio Ambiente, São Paulo.

WINEMILLER, K.O. 1989. Patterns of variation in life history among South American fishes in seasonal environments. Oecologia 81:225-241.

ZAVALA-CAMIN, L.A. 1996. Introdução aos estudos sobre alimentação natural em peixes. Eduem, Maringá. 
